\title{
The effect of carbon monoxide on meiotic maturation of porcine oocytes
}

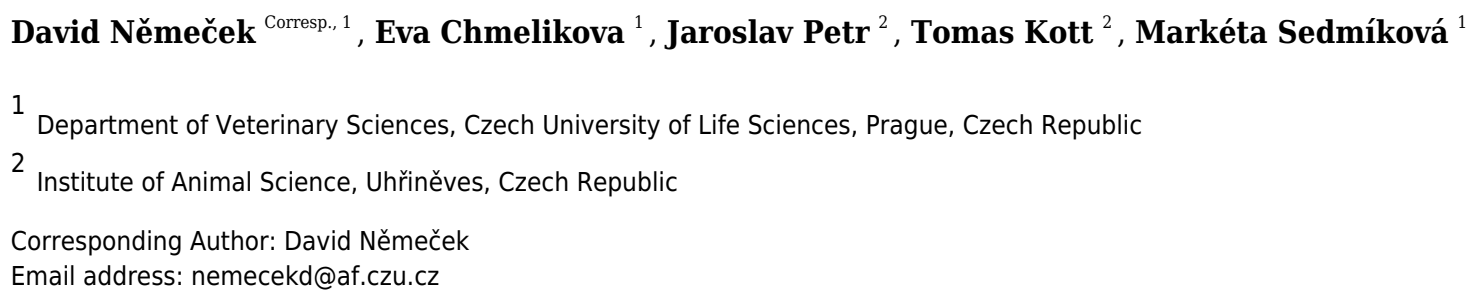

Oxidative stress impairs the correct course of meiotic maturation and it is known that the oocytes are exposed to increased oxidative stress during meiotic maturation in in vitro conditions. Thus, reduction of oxidative stress can lead to improved quality of cultured oocytes. The gasotransmitter carbon monoxide (CO) has a cytoprotective effect in somatic cells. The $\mathrm{CO}$ is produced in cells by the enzyme heme oxygenase (HO) and the heme oxygenase/carbon monoxide ( $\mathrm{HO} / \mathrm{CO}$ ) pathway has been shown to have an antioxidant effect in somatic cells. It has not yet been known whether the CO has an antioxidant effect in oocytes as well. We assessed the level of expression of HO mRNA, using reverse transcription polymerase chain reaction. The HO protein localization was evaluated by the immunocytochemical method. The influence of $\mathrm{CO}$ or $\mathrm{HO}$ inhibition on meiotic maturation was evaluated in oocytes cultured in a culture medium containing CO donor (CORM-2 or CORM-A1) or HO inhibitor Zn-protoporphyrin IX (Zn-PP IX). Detection of reactive oxygen species (ROS) was performed using the oxidant-sensing probe $2^{\prime}, 7^{\prime}$ -

dichlorodihydrofluorescein diacetate. We demonstrated the expression of mRNA and proteins of both $\mathrm{HO}$ isoforms in porcine oocytes during meiotic maturation. The inhibition of HO enzymes by Zn-PP IX did not affect meiotic maturation. CO delivered by CORM-2 or CORM-A1 donors led to a reduction in the level of ROS in the oocytes during meiotic maturation. However, exogenously delivered $\mathrm{CO}$ also inhibited meiotic maturation, especially at higher concentrations. In summary, the CO signaling molecule has antioxidant properties in porcine oocytes and may also be involved in the regulation of meiotic maturation. 


\section{The effect of carbon monoxide on meiotic maturation 2 of porcine oocytes}

4 David Němeček ${ }^{1}$, Eva Chmelikova ${ }^{1}$, Jaroslav Petr ${ }^{2}$, Tomas Kott ${ }^{2}$, Markéta Sedmíková ${ }^{1}$ 5

$6 \quad{ }^{1}$ Department of Veterinary Sciences, Czech University of Life Sciences, Prague, Czech

7 Republic

$8 \quad 2$ Institute of Animal Science, Uhř́něves, Czech Republic

9

10 Corresponding Author:

11 David Němeček ${ }^{1}$

12 Email address: nemecekd@af.czu.cz 
13

14

15

16

17

18

19

20

21

22

23

24

25

26

27

28

29

30

31

32

33

34

35

36

37

38

39

40

41

42

43

44

45

46

47

48

49

50

51

\section{Abstract}

Oxidative stress impairs the correct course of meiotic maturation and it is known that the oocytes are exposed to increased oxidative stress during meiotic maturation in in vitro conditions. Thus, reduction of oxidative stress can lead to improved quality of cultured oocytes. The gasotransmitter carbon monoxide $(\mathrm{CO})$ has a cytoprotective effect in somatic cells. The $\mathrm{CO}$ is produced in cells by the enzyme heme oxygenase (HO) and the heme oxygenase/carbon monoxide ( $\mathrm{HO} / \mathrm{CO})$ pathway has been shown to have an antioxidant effect in somatic cells. It has not yet been known whether the $\mathrm{CO}$ has an antioxidant effect in oocytes as well. We assessed the level of expression of $\mathrm{HO}$ mRNA, using reverse transcription polymerase chain reaction. The $\mathrm{HO}$ protein localization was evaluated by the immunocytochemical method. The influence of $\mathrm{CO}$ or HO inhibition on meiotic maturation was evaluated in oocytes cultured in a culture medium containing CO donor (CORM-2 or CORM-A1) or HO inhibitor Zn-protoporphyrin IX (Zn-PP IX). Detection of reactive oxygen species (ROS) was performed using the oxidant-sensing probe 2',7'-dichlorodihydrofluorescein diacetate. We demonstrated the expression of mRNA and proteins of both $\mathrm{HO}$ isoforms in porcine oocytes during meiotic maturation. The inhibition of HO enzymes by Zn-PP IX did not affect meiotic maturation. CO delivered by CORM-2 or CORM-A1 donors led to a reduction in the level of ROS in the oocytes during meiotic maturation. However, exogenously delivered $\mathrm{CO}$ also inhibited meiotic maturation, especially at higher concentrations. In summary, the CO signaling molecule has antioxidant properties in porcine oocytes and may also be involved in the regulation of meiotic maturation.

\section{Introduction}

Carbon monoxide $(\mathrm{CO})$ is an endogenously produced signaling molecule that affects several cellular functions. $\mathrm{CO}$ is endogenously produced by enzyme heme oxygenase (HO), which catalysis the reduction of heme to biliverdin, trivalent iron, and $\mathrm{CO}$. $\mathrm{HO}$ is known in two isoforms (HO-1 and HO-2) that differ in expression and inducibility. HO-1 is an inducible isoform, and its expression increases after exposure to stressors. HO-2 is a constitutive isoform with basal expression (Maines, 1997; Wu \& Wang, 2005; Ryter \& Choi, 2016). CO produced by $\mathrm{HO}$ enzymes or delivered by $\mathrm{CO}$ donors regulates cytoprotection, the cell cycle, metabolism, and cellular homeostasis (Ryter, Alam \& Choi, 2006; Kolluru et al., 2017). It has been proved that the heme oxygenase/carbon monoxide $(\mathrm{HO} / \mathrm{CO})$ pathway is also involved in the regulation of reproduction. Zenclussen et al. examined the importance of HO-1 in mouse ovaries, and they have shown that HO-1 deficiency in mice reduces oocyte fertilizability (Zenclussen et al., 2012). In our previous publication, we demonstrated that exogenously delivered CO reduced caspase-3 activity and apoptosis in aged porcine oocytes (Němeček et al., 2017). However, it has not yet been investigated whether $\mathrm{CO}$ regulates the level of oxidative stress in oocytes. Proper meiotic maturation in in vitro conditions is crucial for the development of biotechnological methods and the use of assisted reproduction methods. A balanced redox state is important for proper oocyte development (Soto-Herasa \& Paramio, 2020), but in vitro 
52 cultivation systems of oocytes are characterized by increased oxidative stress (Khazaei \& Aghaz, 53 2017).

54 Oxidative stress leads to deterioration of oocyte quality. This can disrupt meiotic maturation and

55

56

57

58

59

60

61

62

63

64

65

66

67

68

69

70

71

72

73

74

75

76

77

78

79

80

81

82

83

84

85

86

87

88

89

90

91

eventually arrested cell cycle and activates apoptosis. Oocytes impaired by oxidative stress have lower fertilization and developmental potential (Agarwal et al., 2012; Prasad et al., 2016; Wang et al., 2017; Xie et al., 2018). Antioxidant supplementation of the culture medium can be used to avoid the harmful effect of oxidative stress. For example, the non-enzymatic antioxidant melatonin has been shown to improve oocyte maturation, fertilization rate, and rate of blastocyst formation (Tamura et al., 2020; Soto-Herasa \& Paramio, 2020). The use of antioxidants can therefore ameliorate in vitro cultivation conditions, but novel substances are still needed to reduce oxidative stress during in vitro oocyte cultivation to improve oocyte quality. CO has already been shown to reduce oxidative stress also in somatic cells (Piantadosi, 2008). Additionally, several factors that control meiotic maturation are simultaneously the cellular target of CO in somatic cells. These factors include MPF, MAPK, JNK2, p38 kinase, and potassium ion channels. Changes in the activity of these factors lead to the alteration of meiotic maturation (Weston \& Davis, 2007; Oh, Han \& Conti, 2010; Huang et al., 2011; Miyagaki et al., 2014; Carvacho et al., 2018). It has already been shown that CO regulates these signaling pathways in somatic cells (Kim, Ryter \& Choi, 2006; Peers et al., 2015; Ryter \& Choi, 2016), but studies on the effect of $\mathrm{CO}$ on oocyte meiotic maturation have been lacking. In somatic cells, CO reduces the expression of cyclins and thereby regulates the cell cycle (Bauer et al., 2016); by activating MKK3/p38 MAPK and NF-kB pathways, CO reduces Fas/Fas ligand interaction, increases expression of anti-apoptotic genes and thus prevents apoptosis (Ryter, Ma \& Choi, 2018; Kim \& Choi, 2018).

For these reasons, we assumed that $\mathrm{CO}$ could reduce oxidative stress in oocytes and thus decrease the effect of negative factors on in vitro meiotic maturation. Therefore, $\mathrm{CO}$ could increase the quality of in vitro matured oocytes. We focused on the $\mathrm{HO} / \mathrm{CO}$ signaling pathway in porcine oocytes during meiotic maturation. Our aim was to determine the effect of the $\mathrm{HO} / \mathrm{CO}$ signaling pathway on porcine oocytes and levels of ROS during their meiotic maturation.

\section{Materials \& Methods}

\section{Porcine oocytes in vitro culture}

Porcine ovaries were obtained from slaughtered prepubertal gilts (Large White $x$ Landrace hybrids, slaughter weight $110 \mathrm{~kg}$ ) that were in an unknown phase of the estrous cycle. Follicular fluid was obtained by aspiration of follicles $(2-5 \mathrm{~mm}$ in diameter) using a syringe with a $20 \mathrm{G}$ needle. Only oocytes with intact cytoplasm and compact cumulus were used for further experiments. Oocytes were cultured in a 4-well multidish (Nunc, Denmark) in modified TCM199 culture medium (Sigma-Aldrich, USA), containing sodium bicarbonate (32.5 mM; SigmaAldrich, USA), calcium L-lactate ( $2.75 \mathrm{mM}$; Sigma-Aldrich, USA), sodium pyruvate ( 0.25 $\mathrm{mg} / \mathrm{ml}$; Sigma-Aldrich, USA), gentamicin ( $0.025 \mathrm{mg} / \mathrm{ml}$; Sigma-Aldrich, USA), HEPES (6.3 $\mathrm{mM}$; Sigma-Aldrich, USA), 10\% (v/v) foetal calf serum (Gibco BRL, Life Technologies,

Peer) reviewing PDF | (2020:08:52279:3:0:NEW 30 Nov 2020) 
92 Germany), and 13.5 IU eCG: 6.6 IU hCG/ml (P.G. 600, Intervet, Boxmeer, Netherlands).

93 Oocytes were cultured to the stage of first (MI) or second meiotic metaphase (MII) for 24 or 48

94 hours, respectively, in $1 \mathrm{ml}$ of modified TCM-199 culture medium $\left(5 \% \mathrm{CO}_{2}, 39^{\circ} \mathrm{C}\right)$.

95 The influence of $\mathrm{CO}$ on meiotic maturation was evaluated in oocytes cultured in a culture

96 medium containing CO donors. We used CO donors CORM-2 (tricarbonyl dichlororuthenium

97 (II) dimer; Sigma-Aldrich, USA) and CORM-A1 (sodium boranocarbonate; Sigma-Aldrich, 98 USA). These donors differ in the kinetics of CO release: CORM-2 is a rapid CO releaser and

99 CORM-A1 is a slow CO releaser. CORM-2, at concentrations of 5.0, 25.0, 50.0, and 100.0 $\mu \mathrm{M}$

100 dissolved in dimethyl sulfoxide (DMSO), or CORM-A1, at 25.0, 50.0 and 100.0 $\mu \mathrm{M}$ dissolved in

101 distilled water, were used. The control group of oocytes was cultured in a culture medium

102 containing inactive CORM-2 (ruthenium (III) chloride; iCORM-2; Sigma-Aldrich, USA) at

103 concentrations of $100.0 \mu \mathrm{M}$ dissolved in DMSO or inactive CORM-A1 (iCORM-A1). iCORM-

104 A1 was obtained by dissolving CORM-A1 $(100.0 \mu \mathrm{M})$ in $0.1 \mathrm{M} \mathrm{HCl}$, dissociating $\mathrm{CO}$, and then

105 neutralizing to $\mathrm{pH}$ 7.4. HO inhibitor Zn-protoporphyrin IX (Zn-PP IX; Sigma-Aldrich, USA), at

106 concentrations of 1.0, 2.5, 5.0, 10.0 and $25.0 \mu \mathrm{M}$ dissolved in DMSO, was used to evaluate the

107 effect of HO inhibition on meiotic maturation. The control group was cultured in a culture

108 medium containing only DMSO at the final concentration of $0.25 \%$. Cultivation in iCORM-2,

109 iCORM-A1, and DSMO did not significantly affect meiotic maturation (Supplementary Table

$110 \mathrm{~S} 1)$.

111

112

113

\section{Reverse Transcription Polymerase Chain Reaction}

114

The presence and amount of HO-1 mRNA and HO-2 mRNA were studied by reverse

115 transcription polymerase chain reaction (RT-PCR). RNA obtained from oocytes was transcribed into cDNA with a High Capacity cDNA Achieve Kit (Applied Biosystems, USA); the final amount was $100 \mu$ l. Based on the knowledge of HO-1 and HO-2 sequences, the specific primers to amplify products were designed (Supplementary Table S2). Standard TaqMan PCR kit protocol was used (Applied Biosystems, USA) for RT-PCR. The reaction ran in a $10 \mu \mathrm{l}$ reaction mixture, containing $500 \mathrm{nM}$ of gene-specific primers, $200 \mathrm{nM}$ $1 \mu 1 \mathrm{cDNA}$ and nuclease-free water. 7500 Fast Real-Time PCR System (Life Technologies, USA) was used for the reaction. Based on the obtained data, the relative amount of mRNA for each isoform was calculated using the $2^{-\Delta \Delta C T}$ arithmetic equation, according to the $\mathrm{Ct}$ method and expressed in comparison to GAPDH as an endogenous control.

125

126

127

\section{Immunocytochemistry}

After completion of the oocyte cultivation period, zona pellucida was removed from oocytes with $0.1 \%$ pronase in phosphate-buffered saline (PBS) solution, and oocytes were fixed in $2.5 \%$ paraformaldehyde in PBS. After membrane permeabilization $(0.5 \%$ Triton X in PBS with $0.01 \%$ bovine serum albumin; BSA), the oocytes were rinsed in $0.1 \%$ Tween 20 in PBS. Incubation with mouse primary monoclonal antibody (anti-heme oxygenase-1 or anti-heme oxygenase-2; 
132 Abnova; Taiwan; 1: 200) was performed overnight (14-16 hours) in 0.1\% BSA and $0.1 \%$ Tween 13320 in PBS at $4{ }^{\circ} \mathrm{C}$. Oocytes were rinsed three times ( $0.1 \%$ Tween 20 in PBS) and incubated in 134 secondary anti-mouse IgG antibody conjugated with fluorescein-5-isothiocyanate (FITC, Sigma135 Aldrich, USA; $1: 100$ ) at room temperature in $0.1 \%$ BSA and $0.1 \%$ Tween 20 in PBS for 1 hour. 136 The specificity of the primary antibodies was confirmed in our previous work by Western blot 137 (Němeček et al., 2017). Chromatin was stained with 4',6-diamidine-2-phenylindole (DAPI; 138 Sigma-Aldrich, USA). To control for secondary antibody non-specific binding detection, a 139 control group of oocytes was cultured in a cultivation medium without the primary antibody. 140 Oocytes were assessed using a confocal scanning microscope (Zeiss, Germany), and intracellular 141 localization was determined based on signal intensity using NIS Elements 3.4 image analysis 142 (Nikon, Japan). Data were expressed relatively as the mean signal intensity of the FITC 143 fluorescence related to the basal signal intensity of the appropriate control group.

144

\section{Assessment of meiotic maturation of oocytes}

146 After a culture period, cumulus cells were denuded by repeated pipetting through a thin-walled glass pipette. Then the oocytes were fixed for at least 24 hours in a solution of ethanol and acetic acid $(3: 1, v / v)$, stained with orcein, and evaluated under a phase-contrast microscope. The stages of meiotic maturation were assessed based on nuclear maturation, as oocytes at germinal vesicle stage (GV; oocytes with visible germinal vesicle), metaphase I (MI; oocytes with chromosomes arranged in metaphase figure), and metaphase II (MII; oocytes with the extruded first polar body). Abnormal oocytes were evaluated as degenerated (Deg).

153

\section{Reactive oxygen species assessment}

155 Production of reactive oxygen species (ROS) was measured in oocytes after 48 hours of culture. After the cultivation period, oocytes were stained with $10 \mu \mathrm{M} 2^{\prime}, 7^{\prime}$-dichlorodihydrofluorescine diacetate (Sigma-Aldrich, USA) for 20 minutes at $39^{\circ} \mathrm{C}$. ROS production in oocytes was evaluated using a confocal scanning microscope (Zeiss, Germany). Images were analyzed using NIS Elements. Data were expressed relatively as mean signal intensity related to the signal

161 intensity of the appropriate control group.

\section{Experimental design}

\section{HO-1 and HO-2 $\mathrm{mRNA}$ and proteins detection}

165

166

167

168

169

170

171
The aim was to assess the expression of HO- 1 and HO-2 mRNA and proteins in porcine oocytes during meiotic maturation. Detection of mRNA and proteins was performed using RT-PCR or immunolocalization, respectively in oocytes at GV, MI, and MII stages. For RT-PCR, each meiotic stage included 50 oocytes in six independent experiments. For the immunocytochemical method, localization of each isoform was performed on 25 oocytes for each meiotic stage in three independent experiments.

\section{The effect of HO inhibition}


172 The aim was to assess the effect of $\mathrm{HO}$ inhibition on meiotic maturation. Nuclear maturation was

173 evaluated after 48 hours of oocyte cultivation in a culture medium containing Zn-PP. Each

174 concentration of Zn-PP IX included 80 oocytes in three independent experiments.

175

176

The effect of $\mathrm{CO}$ donor

177 The purpose was to determine the effect of $\mathrm{CO}$ donor on meiotic maturation of porcine oocytes.

178 As in the previous experiment, nuclear maturation was evaluated after 48 hours of oocyte

179 cultivation in a culture medium containing CORM-2 or CORM-A1 donors. Each concentration

180 of CORM-2 or CORM-A included 80 oocytes in three independent experiments.

181

182

The effect of $\mathrm{CO}$ donor on ROS production

183

The aim was to assess the effect of CO donor on the production of ROS in porcine oocytes

184

during meiotic maturation. Due to the similar effect of CORM-2 and CORM-A1 found in the

185 previous experiment, only CORM-2 was used. The amount of ROS was measured by

186 immunolocalization in oocytes cultured for 48 hours in a culture medium containing CORM-2.

187 Each concentration of CORM- 2 included 30 oocytes in three independent experiments.

188

189

190

\section{Statistical data analysis}

The data are presented as the mean \pm SEM of at least three independent experiments. The data

191 were statistically evaluated in the STATISTICA 12 program (Statsoft, USA). Statistically

192 significant differences between groups were assessed by analysis of variance (ANOVA) and

193 multiple comparisons using Scheffé's method. A P-value of less than 0.05 was considered to be statistically significant.

195

196

197

198

199

200

201

202

203

204

\section{Results}

\section{HO-1 and HO-2 mRNA and proteins were detected in porcine oocytes}

We have detected HO-1 and HO-2 mRNA in porcine oocytes during meiotic maturation by RTPCR. The difference in HO-1 and HO-2 mRNA levels between oocyte categories (GV, MI, and MII) was not statistically significant (Table 1 ).

We evaluated the expression of HO-1 and HO-2 proteins in porcine oocytes during meiotic maturation (GV, MI, and MII). Using the immunocytochemical method, we demonstrated the presence of both $\mathrm{HO}$ isoforms at all stages of meiotic maturation (GV, MI, MII) (Figure 1). The localization of the HO-1 isoform was dependent on the stage of meiotic maturation. In oocytes at the GV stage, HO-1 was localized primarily in the germinal vesicle; in the case of oocytes at MI and MII stage, HO-1 was detected in the perichromosomal region. The expression level of HO-1 was significantly lower in the cytoplasmic region than in the perichromosomal area (Figure 1). In the case of the HO-2 isoform, we did not find a significant difference in the level of expression 209 between the cytoplasmic and perichromosomal regions in oocytes at MI and MII stages. In 210 oocytes at the GV stage, HO-2 expression was significantly lower in the germinal vesicle than in 
211 the cytoplasmic region. We did not find significant changes in the overall level of expression of

212 HO-1 and HO-2 during meiotic maturation (Figure 2).

213

214

215

Inhibition of HO enzymes did not affect meiotic maturation

216

We evaluated the effect of $\mathrm{HO}$ inhibition on meiotic maturation by assessing nuclear maturation

217 in oocytes cultured in a culture medium containing HO inhibitor Zn-PP IX. The cultivation of

218 oocytes in a culture medium containing Zn-PP IX did not significantly affect meiotic maturation,

219 and the proportion of matured oocytes did not differ between the control and experimental

220 groups (99.2 $\pm 0.5 \%$ vs $96.4 \pm 2.3 \%-100.0 \pm 0.0 \%$ for control and Zn-PP-IX, respectively). The inhibitor slightly affected nuclear maturation only at the concentration of $5.0 \mu \mathrm{M}$, reducing the

221 proportion of oocytes matured to the MII stage by $4.2 \%$, as compared to the control group (99.2

222 $\pm 0.5 \%$ vs $95.0 \pm 1.8$ for control and Zn-PP-IX, respectively) (Figure 3).

223

224

\section{Carbon monoxide inhibits the meiotic maturation of porcine oocytes}

225

226

By assessing the effect of a $\mathrm{CO}$ donor on oocyte nuclear maturation, we found that the $\mathrm{CO}$ donor inhibited the nuclear maturation of cultured oocytes. Cultivation in a culture medium containing

227

228 CORM-2 or CORM-A1 decreases the proportion of oocytes matured to the MII stage. After the

229 oocytes cultivation in a culture medium containing CORM-2 or CORM-A1, CO donors inhibited

230 nuclear maturation at all used concentrations. Meiotic maturation in media containing CORM-2

231 resulted in a significant reduction of the proportion of oocytes matured to MII ( $85.5 \pm 1.1 \%$ vs $55.1 \pm 1.9-67.9 \pm 1.4 \%$ for control and CORM-2, respectively). CORM-2 at the concentration of $100.0 \mu \mathrm{M}$ had the most pronounced effect on the proportion of oocytes matured to the MII

234 stage. After cultivation in a culture medium containing CORM-2, CO arrested meiotic maturation at the MI stage and significantly increased the proportion of oocytes at the MI stage ( $7.8 \pm 0.7 \%$ vs $16.1 \pm 2.0 \%-36.5 \% \pm 2.9$ for control and CORM- 2 , respectively). The effect was dose-dependent. CORM-2 at a concentration of $100.0 \mu \mathrm{M}$ had the most potent inhibitory effect on meiotic maturation (most significant increase of the proportion of oocytes at the MI stage) (Figure 4A). Just like in the case of CORM-2, cultivation in a medium containing CORM-A1 resulted in a significant reduction of the proportion of oocytes matured to the MII stage $(82.0 \pm 0.5 \%$ vs 45.5 $\pm 2.5 \%-61.4 \pm 2.5 \%$ for control and CORM-A1, respectively). Also, CORM-A1 at the concentration of $100.0 \mu \mathrm{M}$ had the most pronounced effect on the proportion of oocytes matured to the MII stage. Meiotic maturation was arrested mainly in the MI stage $(6.9 \pm 1.1 \%$ vs $16.5 \pm$ $1.3 \%-29.9 \pm 1.9 \%$ for control and CORM-A1, respectively). Furthermore, oocyte cultivation in a culture medium containing CORM-A1 at the concentration of $25.0 \mu \mathrm{M}$ also increased the proportion of oocytes at the GV stage (5.6 $\pm 1.4 \%$ vs $14.5 \pm 1.3 \%$ for control and CORM-A1, respectively) (Figure 4B).

248 After analysis of oocytes cultured for 72 hours in a culture medium containing CORM-2, we 249 found that the oocytes did not complete meiotic maturation to the MII stage $(80.5 \pm 0.7 \%$ vs 42.4 250 $\pm 2.1 \%-58.2 \pm 1.4 \%$ for control and CORM-2, respectively) and meiotic maturation remained 
251

252

253

254

255

256

257

258

259

260

261

262

263

264

265

266

267

268

269

270

271

272

273

274

275

276

277

278

279

280

281

282

283

284

285

286

287

288

289

290

arrested mainly at the MI stage ( $5.8 \pm 1.0 \%$ vs $24.5 \pm 2.0 \%-40.5 \% \pm 1.9$ for control and CORM-2, respectively). The COMR-2 had the most significant effect at the concentrations of $50.0 \mu \mathrm{M}$ and $100.0 \mu \mathrm{M}$. (Figure 5).

\section{Carbon monoxide reduces the production of reactive oxygen species in porcine oocytes during meiotic maturation}

We found that after meiotic maturation in a culture medium containing CO donor CORM-2, the amount of ROS in porcine oocytes decreased (Figure 6).

Oocytes cultivated for 48 hours in a culture medium containing CORM-2 led to a significant decrease in ROS production. All used CORM-2 concentrations had a significant effect on ROS level reduction. After meiotic maturation in a culture medium containing CORM-2 at 5.0, 25.0, 50.0 , or $100.0 \mu \mathrm{M}$, the amount of ROS was reduced by $29.1 \%, 47.0 \%, 46.3 \%$, and $48.9 \%$, respectively. The effect of CORM-2 at the concentrations $25.0,50.0$, and $100.0 \mu \mathrm{M}$ was not significant (Figure 7).

\section{Discussion}

In the present work, we studied the importance of $\mathrm{HO} / \mathrm{CO}$ for the meiotic maturation of porcine oocytes. Both heme oxygenase isoforms catalyze the oxidative degradation of heme. An excess of the heme molecule in the cell causes oxidative stress, and its removal by HO activity is important for cell protection (Chiabrando et al., 2014). During ovulation, the amount of heme in the ovaries increases, and it is believed that the enzyme HO protects the ovarian cells from the degradation of the heme molecule (Zenclussen et al., 2012). Degradation of heme by HO enzymes produces $\mathrm{CO}$, a molecule with two faces. At high concentrations, $\mathrm{CO}$ has several toxic properties that are well known. However, at low concentrations, $\mathrm{CO}$ is an important signaling molecule that has cytoprotective, antiapoptotic, and antioxidative properties (Ryter \& Choi, 2016; Kolluru et al., 2017). Considering the effects of $\mathrm{HO} / \mathrm{CO}$ in somatic cells, we assume that $\mathrm{CO}$ could contribute to the protection of oocytes during meiotic maturation, especially in in vitro conditions. Our previous work demonstrated the presence of $\mathrm{HO}$ in oocytes exposed to in vitro aging (Němeček, Dvoř́ková \& Sedmíková, 2017). In the present study, we evaluated the HO expression during meiotic maturation. We have not only proved the occurrence of both $\mathrm{HO}$ mRNA and proteins in porcine oocytes during their meiotic maturation, but we have also shown that $\mathrm{CO}$ has antioxidative properties in porcine oocytes. However, $\mathrm{CO}$ impairs meiotic maturation, particularly at high concentrations.

Cellular localization of the HO-1 isoform in oocytes predominated in the perichromosomal region, both in oocytes at the GV stage and in oocytes at the MI or MII stage. Localization of HO-1 was prevalent in the perichromosomal area also in aged porcine oocytes (Němeček et al., 2017) and in the nucleus of bovine granulosa cells (Wang et al., 2018). In somatic cells, HO-1 was mainly localized in the endoplasmic reticulum (Dennery, 2014), and translocation of HO-1 to the nuclear region may occur in response to stress factors (Lin et al., 2007). In the nuclear area, $\mathrm{HO}-1$ regulates the activity of transcription factors such as AP-1 and $\mathrm{NrF} 2$, which increase 
291

292

293

294

295

296

297

298

299

300

301

302

303

304

305

306

307

308

309

310

311

312

313

314

315

316

317

318

319

320

321

322

323

324

325

326

327

328

329

330

the resistance of cells to oxidative stress (Li Volti et al., 2004; Lin et al., 2007; Biswas et al., 2014; Tibullo et al., 2016). It is shown, for example, that nuclear HO-1 regulates the expression of antioxidative enzymes $\gamma$-glutamylcysteine synthetase, glutathione peroxidase, catalase, and methionine sulfoxide reductase. These enzymes are essential for resistance against oxidative stress (Collinson et al., 2010), and they are involved in the regulation of oxidative stress also in oocytes (Cetica et al., 2001). In addition to oxidative stress regulation in somatic cells, the $\mathrm{HO} / \mathrm{CO}$ system also influences the cell cycle. Nuclear HO-1 may regulate the initiation of meiotic maturation through activation of the transcription factor Nrf2. It is already known that nuclear HO-1 regulates the activity of transcription factors (Nrf2 and AP-1) in somatic cells ( $L i$ Volti et al., 2004; Lin et al., 2007; Biswas et al., 2014; Tibullo et al., 2016). Qiu and Yao suggest that $\mathrm{Nrf2}$ is involved in the initiation of meiosis since the inhibition of Nrf2 results in altered expression of the cell cycle-related genes and delayed progression in leptotene ( $Q i u \& Y a o$, 2017). For these reasons, nuclear HO-1 may regulate the activity of transcription factors and, thereby, meiotic maturation. In contrast to HO-1, the HO-2 isoform predominated in the cytoplasmic region. In somatic cells, HO-2 occurs mainly in the cytoplasm as a membrane protein of the endoplasmic reticulum (Ma et al., 2004; Linnenbaum et al., 2012). The HO-2 isoform is a constitutively active enzyme that does not respond to activation by stress factors. Also, in the porcine oocytes, we did not observe significant changes in HO-2 expression. It is believed that the HO-2 is responsible for the stable production of $\mathrm{CO}$ and thus can form a barrier against cellular damage, e.g., by protecting against the negative effect of radicals derived from cellular metabolism (Muñoz-Sánchez \& Chánez-Cárdenas, 2014). For these reasons, HO-2 could have a protective function also in oocytes. However, we have shown that inhibition of both $\mathrm{HO}$ isoforms does not significantly affect meiotic maturation. Though in the case of HO- 1 , it is proven that both enzymatically active HO-1 protein and HO-1 protein with reduced enzymatic activity have antioxidative properties. This effect is probably due to the binding of inactive HO-1 to other proteins, such as transcription factors. Thus, HO-1 affects the transcription factors AP-1 and Nrf2 (Lin et al., 2007; Dennery, 2014) due to the localization of HO-1 in the perichromosomal region of porcine oocytes. For these reasons, HO-1 could affect the protein's activity despite the presence of an inhibitor. To assess the significance of HO during meiotic maturation, it would be useful, for example, to study the effect in animals that are deficient in $\mathrm{HO}$ enzymes. Zenclussen et al. examined the effect of HO-1 gene deficiency on oocyte ovulation, fertilization, and corpus luteum maintenance in mice. It is not known whether the HO1 deficiency directly affected meiotic maturation, but the fertilization rate of oocytes from HO-1 deficient animals was decreased (Zenclussen et al., 2012).

The gasotransmitter $\mathrm{CO}$ is already well known for its cytoprotective properties and also for the beneficial effect the exogenous delivery of CO can have on cells (Wegiel, Chin \& Otterbein, 2008). Our results showed that cultivation in a culture medium containing $\mathrm{CO}$ donors led to a reduction in the level of ROS in porcine oocytes. At the same time, however, $\mathrm{CO}$ arrested meiotic maturation, particularly at the MI stage. This effect was dose-dependent. It was shown in somatic cells that $\mathrm{CO}$ has an antioxidative effect and reduces the amount of ROS (Pileggi et al., 
331

332

333

334

335

336

337

338

339

340

341

342

343

344

345

346

347

348

349

350

351

352

353

354

355

356

357

358

359

360

361

362

363

364

365

366

367

368

369

370

2001; Brouard et al., 2002; Motterlini \& Otterbein, 2010; Li et al., 2016). CO is also involved in cell cycle regulation (Wegiel, Chin \& Otterbein, 2008). In oocytes, ROS negatively affects meiotic maturation, fertilization, and developmental competence. Protection against oxidative stress is important, and cultivation in a culture media containing antioxidants improves meiotic maturation (Combelles, Gupta \& Agarwal, 2009; Prasad et al., 2016). For these reasons, we consider the ability of $\mathrm{CO}$ to reduce the amount of ROS during meiotic maturation to be beneficial. The different concentrations did not have significantly different effects, and even the low concentration of CO led to a significant reduction in ROS levels. In aging oocytes, $\mathrm{CO}$ reduces caspase- 3 activity and the occurrence of negative effects of postovulatory aging (Němeček et al., 2017). ROS are mainly responsible for the adverse effects of postovulatory oocyte aging; therefore, reducing the level of ROS may lead to improved oocyte quality and developmental potential (Lord \& John Aitken, 2013; Prasad et al., 2016). The effect of CO on ROS levels has not yet been studied in aged oocytes, but because we proved the antioxidant effect of $\mathrm{CO}$ in oocytes, we assume that $\mathrm{CO}$ reduced the negative effects of aging by reducing the level of ROS. Thus, $\mathrm{CO}$ could have a beneficial effect during meiotic maturation and postovulatory aging of oocytes. However, ROS does not only have a negative effect on cells. It is also demonstrated that ROS is involved in the regulation of meiotic maturation and that a low level of ROS is essential for proper meiotic maturation (Soto-Herasa \& Paramio, 2020). Oocyte cultivation in a medium containing high concentrations of ROS scavengers can lead to the inhibition of meiotic maturation (Tiwari et al., 2016). For example, supplementation of the culture medium with the non-enzymatic antioxidants ascorbic acid and 3-tert-butyl-4hydroxyanisole leads to the inhibition of meiotic maturation (Khazaei \& Aghaz, 2017). In porcine oocytes, a high CO concentration could lead to a significant reduction of ROS level and alteration of the oxidative balance. Furthermore, a high concentration of $\mathrm{CO}$ can have a detrimental effect on meiotic maturation: meiotic maturation arrest. Therefore, we suggest that CO may be beneficial in low concentrations in in vitro oocyte culture.

The oocyte is not isolated in the cumulus-oocyte complex, but granulosa cells are important for its successful development. Granulosa cells supply nutrients and metabolites through gap junctions to oocytes and secrete paracrine signals to regulate oocytes. Oocyte also lacks several defense mechanisms that are provided by the granulosa cells. On the other hand, oocytes regulate granulosa cell proliferation and differentiation (Alam \& Miyano, 2020; von Mengden, Klamt \& Smitz, 2020). Both HO isoforms are detected in granulosa cell (Alexandreanu \& Lawson, 2003; Bergandi et al., 2014; Wang et al., 2018; Wang et al., 2019). Particularly inducible HO-1 is involved in protecting granulosa cells against stressors. They are protected against oxidative stress via the Nrf2/HO-1 pathway (Wang et al., 2018). Increased stress leads to the induction of HO-1 expression and thus an increase in CO production in granulosa cells (Wang et al., 2018; Wang et al., 2019). The level of stress may be too high, and the abilities of endogenous HO-1 may be overwhelmed. This then leads to cell damage. Significantly increased levels of HO-1 expression in granulosa cells may be associated with impaired oocyte competence (Bergandi et al., 2014), suggesting that an excessive ROS production can trigger oocyte damage (Canosa et 
371 al., 2020). The addition of protective agents, such as antioxidants, can then prevent cell damage. 372 Antioxidant melatonin has been shown to enhance oocyte and embryo quality (Rizzo, Raffone \& 373 Benedetto, 2010). In granulosa cells, melatonin increases the expression of HO-1 and protects 374 them from oxidative stress ( $Y$ u et al., 2019). Also, the induction of HO-1 activity by hemin 375 increases antioxidant defenses and attenuates ROS generation and apoptosis in stressed 376 granulosa cells (Wang et al., 2019). CO is mainly responsible for this protective effect. The 377 addition of $\mathrm{CO}$ through the CORM-2 demonstrates that $\mathrm{CO}$ regulates the apoptosis of granulosa 378 cells through the ERK1/2 pathway (Wang et al., 2019). We assume that CO may affect meiotic

379

380

381

382

383

384

385

386

387

388

389

390

391

392

393

394

395

396

397

398

399

400

401

402

403

404

405

406

407

408

409

410 maturation also by affecting signaling pathways in granulosa cells. For example, the ERK1/2 pathway is important for the resumption of meiotic maturation (Shimada, 2012). On the other hand, $\mathrm{CO}$ is an activator of guanylyl cyclase. In the follicle cGMP is synthesized by guanylyl cyclase in granulosa cells and diffuses to the oocyte to inhibit the hydrolysis activity of PDE3A on cAMP, ultimately maintains the oocyte meiotic arrest (Shuhaibar et al., 2015; Jaffe \& Egbert, 2017). The CO effect is probably complex and affects granulosa cells, oocytes, and their communication during meiotic maturation.

Meiotic maturation in in vitro conditions may result in oocytes with asynchronous nuclear and cytoplasmic maturation. This is important because adequate oocyte developmental competence requires synchronization between nuclear and cytoplasmic maturation. This condition decreases oocyte fertilizability and impairs early embryonic development (Ali \& Miron, 2006; Rybska et al., 2018; Leal et al., 2018). Synchronization techniques based on the use of reversible meiotic inhibitors can prevent this asynchrony. The purpose of this inhibition is to temporarily block meiotic progression during maturation. The block is then removed to allow the oocytes to mature under in vitro conditions (Vanhoutte et al., 2009; Gil et al., 2017; Leal et al., 2018). For example, temporarily arresting meiotic maturation by phosphodiesterase 3-inhibitor leads to an increase in oocyte quality and developmental potential (Vanhoutte et al., 2009; Gil et al., 2017; Leal et al., 2018). It would be an interesting question whether the application of CO to the culture system could lead to the synchronization of nuclear and cytoplasmic maturation. This could improve the quality of in vitro cultured oocytes.

Other signaling pathways may be responsible for the inhibition of meiotic maturation caused by $\mathrm{CO}$, such as the JNK kinase-signaling pathway. In oocytes, the inhibition of JNK arrests meiotic maturation (Huang et al., 2011) also, it is shown that CO inhibits JNK activity in somatic cells (Kim, Ryter \& Choi, 2006). CO can also regulate meiotic maturation through interaction with other gasotransmitters. It has already been shown that nitric oxide (NO) and hydrogen sulfide $\left(\mathrm{H}_{2} \mathrm{~S}\right.$ ) regulate meiotic maturation (Bu et al., 2003; Nevoral et al., 2014). In the case of $\mathrm{H}_{2} \mathrm{~S}$, it has been shown to accelerate the meiotic maturation of oocytes (Nevoral et al., 2014, 2015). CO inhibits the activity of $\mathrm{H}_{2} \mathrm{~S}$ producing enzymes and thereby decreases $\mathrm{H}_{2} \mathrm{~S}$ production (Giuffrè \& Vicente, 2018). Inhibition of $\mathrm{H}_{2} \mathrm{~S}$ producing enzymes by inhibitors leads to impaired meiotic maturation of porcine oocytes (Nevoral et al., 2015). In somatic cells, it has been shown that $\mathrm{CO}$ can reduce the level of cyclin proteins (Bauer et al., 2016); for example, MPF is an enzymatic complex composed of cyclin-dependent kinase 1 and cyclin B (Kishimoto, 2018). Furthermore, 
411 MPF activity is crucial for meiotic maturation, catalyzing entry into the M-phase of meiosis I and 412 meiosis II (Schmitt \& Nebreda, 2002). If CO affects cyclin B levels, then it could regulate MPF 413 activity in this way.

414

415

416

417

418

419

420

421

422

423

424

425

426

427

428

429

430

431

432

433

434

435

436

437

438

439

440

441

442

443

444

445

446

447

448

449

450

\section{Conclusions}

In summary, our work has shown that $\mathrm{CO}$ inhibits meiotic maturation and reduces ROS production in porcine oocytes. We assume that in oocytes, $\mathrm{HO} / \mathrm{CO}$ may regulate the oxidative state and contributes to the protection against oxidative stress. Furthermore, we assume that $\mathrm{CO}$ probably affects some of the signaling pathways that regulate meiotic maturation. This leads to inhibition of meiotic maturation. To further assess the effect of $\mathrm{CO}$ during meiotic maturation, it is necessary to focus on the mechanism by which $\mathrm{CO}$ regulates meiotic maturation. We conclude that the $\mathrm{HO} / \mathrm{CO}$ signaling pathway is an unexplored part that regulates meiotic maturation and that also regulates oxidative stress in oocytes.

\section{References}

Agarwal A, Aponte-Mellado A, Premkumar BJ, Shaman A, Gupta S. 2012. The effects of oxidative stress on female reproduction: a review. Reproductive Biology and Endocrinology, 10: 49. DOI: 10.1186/1477-7827-10-49.

Alam MH, Miyano T. 2020. Interaction between growing oocytes and granulosa cells in vitro. Reproductive Medicine and Biology, 19: 13-23. DOI: 10.1002/rmb2.12292.

Alexandreanu IC, Lawson DM. 2003. Heme oxygenase in the rat ovary: immunohistochemical localization and possible role in steroidogenesis. Experimental Biology and Medicine, 228: 59-63. DOI: 10.1177/153537020322800108.

Bauer A, Mylroie H, Thornton CC, Calay D, Birdsey GM, Kiprianos AP, Wilson GK, Soares MP, Yin X, Mayr M, Randi AM, Mason JC. 2016. Identification of cyclins A1, E1 and vimentin as downstream targets of heme oxygenase-1 in vascular endothelial growth factormediated angiogenesis. Scientific Reports, 6. DOI: 10.1038/srep29417.

Bergandi L, Basso G, Evangelista F, Canosa S, Dalmasso P, Aldieri E, Revelli A, Benedetto Ch, Ghigo D. 2014. Inducible nitric oxide synthase and heme oxygenase 1 are expressed in human cumulus cells and may be used as biomarkers of oocyte competence. Reproductive Sciences, 21: 1370-1377. DOI: 10.1177/1933719114525268.

Biswas C, Shah N, Muthu M, La P, Fernando AP, Sengupta S, Yang G, Dennery PA. 2014. Nuclear heme oxygenase-1 (HO-1) modulates subcellular distribution and activation of Nrf2, impacting metabolic and anti-oxidant defenses. Journal of Biological Chemistry, 289: 26882-26894. DOI: 10.1074/jbc.M114.567685.

Brouard S, Berberat PO, Tobiasch E, Seldon MP, Bach FH, Soares MP. 2002. Heme oxygenase1-derived carbon monoxide requires the activation of transcription factor NF- $\mathrm{BB}$ to protect endothelial cells from tumor necrosis factor- $\alpha$-mediated apoptosis. Journal of Biological Chemistry, 277: 17950-17961. DOI: 10.1074/jbc.M108317200.

Bu S, Xia G, Tao Y, Lei L, Zhou B. 2003. Dual effects of nitric oxide on meiotic maturation of 
451

452

453

454

455

456

457

458

459

460

461

462

463

464

465

466

467

468

469

470

471

472

473

474

475

476

477

478

479

480

481

482

483

484

485

486

487

488

489

490

mouse cumulus cell-enclosed oocytes in vitro. Molecular and Cellular Endocrinology, 207: 21-30. DOI: 10.1016/S0303-7207(03)00213-2.

Canosa S, Bergandi L, Macrí Ch, Charrier L, Paschero C, Carosso A, Di Segni N, Silvagno F, Gennarelli G, Benedetto Ch, Revelli A. 2020. Morphokinetic analysis of cleavage stage embryos and assessment of specific gene expression in cumulus cells independently predict human embryo development to expanded blastocyst: a preliminary study. Journal of Assisted Reproduction and Genetics, 37: 1409-1420. DOI: 10.1007/s10815-020-01806-6.

Carvacho I, Piesche M, Maier TJ, Machaca K. 2018. Ion channel function during oocyte maturation and fertilization. Frontiers in Cell and Developmental Biology, 6. DOI: 10.3389/fcell.2018.00063.

Cetica PD, Pintos LN, Dalvit GC, Beconi MT. 2001. Antioxidant enzyme activity and oxidative stress in bovine oocyte in vitro maturation. IUBMB Life, 51: 67-64. DOI: 10.1080/15216540152035073.

Chiabrando D, Vinchi F, Fiorito V, Mercurio S, Tolosano E. 2014. Heme in pathophysiology: A matter of scavenging, metabolism and trafficking across cell membranes. Frontiers in Pharmacology, 5. DOI: 10.3389/fphar.2014.00061.

Collinson EJ, Wimmer-Kleikamp S, Gerega SK, Yang YH, Parish CR, Dawes IW, Stocker R. 2010. Heme Oxygenase-1 Affords Cellular Antioxidant Protection via the Transcriptional Regulation of Known Antioxidant Genes. Journal of Biological Chemistry, 286: 2205-2214. DOI: $10.1074 /$ jbc.M110.187062.

Combelles CMH, Gupta S, Agarwal A. 2009. Could oxidative stress influence the in-vitro maturation of oocytes? Reproductive BioMedicine Online, 18: 864-880. DOI: 10.1016/S1472-6483(10)60038-7.

Dennery PA. 2014. Signaling function of heme oxygenase proteins. Antioxidants and Redox Signaling, 20: 1743-1753. DOI: 10.1089/ars.2013.5674.

Gil MA, Nohalez A, Martinez CA, Ake-Villianueva JR, Centurion-Castro F, Maside C, Cuello C, Roca J, Parrilla I, Martinez EA. 2017. Effects of meiotic inhibitors and gonadotrophins on porcine oocytes in vitro maturation, fertilization and development. Reproduction in Domestic Animals, 52: 873-880. DOI: 10.1111/rda.12993.

Giuffrè A, Vicente JB. 2018. Hydrogen sulfide biochemistry and interplay with other gaseous mediators in mammalian physiology. Oxidative Medicine and Cellular Longevity, 2018: 2018:6290931. DOI: 10.1155/2018/6290931.

Huang X, Tong JS, Wang ZB, Yang CR, Qi ST, Guo L, Ouyang YC, Quan S, Sun QY, Qi ZQ, Huang RX, Wang HL. 2011. JNK2 participates in spindle assembly during mouse oocyte meiotic maturation. Microscopy and Microanalysis, 17: 197-205. DOI: $10.1017 /$ S1431927610094456.

Jaffe LA, Egbert JR. 2017. Regulation of mammalian oocyte meiosis by intercellular communication within the ovarian follicle. Annual Review of Physiology, 79: 237-260. DOI: 10.1146/annurev-physiol-022516-034102.

Khazaei M, Aghaz F. 2017. Reactive oxygen species generation and use of antioxidants during 
491

492

493

494

495

496

497

498

499

500

501

502

503

504

505

506

507

508

509

510

511

512

513

514

515

516

517

518

519

520

521

522

523

524

525

526

527

528

529

530

in vitro maturation of oocytes. International Journal of Fertility and Sterility, 11: 63-70. DOI: $10.22074 /$ ijfs.2017.4995.

Kim HP, Ryter SW, Choi AMK. 2006. CO AS A CELLULAR SIGNALING MOLECULE. Annual Review of Pharmacology and Toxicology, 46: 411-449. DOI:

10.1146/annurev.pharmtox.46.120604.141053.

Kim H, Choi S. 2018. Therapeutic aspects of carbon monoxide in cardiovascular disease. International Journal of Molecular Sciences, 19: 2381. DOI: 10.3390/ijms 19082381.

Kishimoto T. 2018. MPF-based meiotic cell cycle control: Half a century of lessons from starfish oocytes. Proceedings of the Japan Academy Series B: Physical and Biological Sciences, 94: 180-203. DOI: 10.2183/pjab.94.013.

Kolluru GK, Shen X, Yuan S, Kevil CG. 2017. Gasotransmitter heterocellular signaling. Antioxidants and Redox Signaling, 26: 936-960. DOI: 10.1089/ars.2016.6909.

Leal GR, Monteiro CAS, Souza-Fabjan JMG, Vasconcelos CO, Nogueira LAG, Ferreira AMR, Serapião RV. 2018. Role of cAMP modulator supplementations during oocyte in vitro maturation in domestic animals. Animal Reproduction Science, 199: 1-14. DOI: 10.1016/j.anireprosci.2018.11.002.

Li Volti G, Ientile R, Abraham NG, Vanella A, Cannavò G, Mazza F, Currò M, Raciti G, Avola $\mathrm{R}$, Campisi A. 2004. Immunocytochemical localization and expression of heme oxygenase1 in primary astroglial cell cultures during differentiation: Effect of glutamate. Biochemical and Biophysical Research Communications, 315: 517-524. DOI: 10.1016/j.bbrc.2004.01.090.

Li C, Zhang C, Wang T, Xuan J, Su C, Wang Y. 2016. Heme oxygenase 1 induction protects myocardiac cells against hypoxia/reoxygenation-induced apoptosis: The role of JNK/cJun/Caspase-3 inhibition and Akt signaling enhancement. Herz, 41: 715-724. DOI: 10.1007/s00059-016-4424-6.

Lin Q, Weis S, Yang G, Weng YH, Helston R, Rish K, Smith A, Bordner J, Polte T, Gaunitz F, Dennery PA. 2007. Heme oxygenase-1 protein localizes to the nucleus and activates transcription factors important in oxidative stress. Journal of Biological Chemistry, 282: 20621-20633. DOI: 10.1074/jbc.M607954200.

Linnenbaum M, Busker M, Kraehling JR, Behrends S. 2012. Heme oxygenase isoforms differ in their subcellular trafficking during hypoxia and are differentially modulated by cytochrome P450 reductase. PLoS ONE, 7: e35483. DOI: 10.1371/journal.pone.0035483.

Lord T, John Aitken R. 2013. Oxidative stress and ageing of the post-ovulatory oocyte. Reproduction, 146: R217-R227. DOI: 10.1530/REP-13-0111.

Ma N, Ding X, Doi M, Izumi N, Semba R. 2004. Cellular and subcellular localization of heme oxygenase-2 in monkey retina. Journal of Neurocytology, 33: 47-415. DOI: 10.1023/B:NEUR.0000046571.90786.6e.

Maines MD. 1997. The heme oxygenase system: A regulator of second messenger gases. Annual Review of Pharmacology and Toxicology, 37: 517-554. DOI: 10.1146/annurev.pharmtox.37.1.517.

Peer) reviewing PDF | (2020:08:52279:3:0:NEW 30 Nov 2020) 
531

532

533

534

535

536

537

538

539

540

541

542

543

544

545

546

547

548

549

550

551

552

553

554

555

556

557

558

559

560

561

562

563

564

565

566

567

568

569

570

von Mengden L, Klamt F, Smitz J. 2020. Redox biology of human cumulus cells: basic concepts, impact on oocyte quality, and potential clinical use. Antioxidants \& Redox Signaling, 32: 522-535. DOI: 10.1089/ars.2019.7984.

Miyagaki Y, Kanemori Y, Tanaka F, Baba T. 2014. Possible Role of p38 MAPK-MNK1-EMI2 Cascade in Metaphase-II Arrest of Mouse Oocytes1. Biology of Reproduction, 91: 1-8. DOI: 10.1095/biolreprod.113.116962.

Motterlini R, Otterbein LE. 2010. The therapeutic potential of carbon monoxide. Nature Reviews Drug Discovery, 9: 728-743. DOI: 10.1038/nrd3228.

Muñoz-Sánchez J, Chánez-Cárdenas ME. 2014. A review on hemeoxygenase-2: Focus on cellular protection and oxygen response. Oxidative Medicine and Cellular Longevity, 2014: 604981. DOI: 10.1155/2014/604981.

Němeček D, Dvořáková M, Heroutová I, Chmelíková E, Sedmíková M. 2017. Anti-apoptotic properties of carbon monoxide in porcine oocyte during in vitro aging. PeerJ, 5: e3876. DOI: $10.7717 /$ peerj.3876.

Němeček D, Dvořáková M, Sedmíková M. 2017. Heme oxygenase/carbon monoxide in the female reproductive system: An overlooked signalling pathway. International Journal of Biochemistry and Molecular Biology, 8: 1-12.

Nevoral J, Petr J, Gelaude A, Bodart JF, Kucerova-Chrpova V, Sedmikova M, Krejcova T, Kolbabova T, Dvorakova M, Vyskocilova A, Weingartova I, Krivohlavkova L, Zalmanova T, Jilek F. 2014. Dual effects of hydrogen sulfide donor on meiosis and cumulus expansion of porcine cumulus-oocyte complexes. PLoS ONE, 9: e99613. DOI: 10.1371/journal.pone.0099613.

Nevoral J, Žalmanová T, Zámostná K, Kott T, Kučerová-Chrpová V, Bodart JF, Gelaude A, Procházka R, Orsák M, Šulc M, Klein P, Dvořáková M, Weingartová I, Víghová A, Hošková K, Krejčová T, Jílek F, Petr J. 2015. Endogenously produced hydrogen sulfide is involved in porcine oocyte maturation in vitro. Nitric Oxide - Biology and Chemistry, 51: 24-35. DOI: 10.1016/j.niox.2015.09.007.

Oh JS, Han SJ, Conti M. 2010. Wee1B, Myt1, and Cdc25 function in distinct compartments of the mouse oocyte to control meiotic resumption. Journal of Cell Biology, 188: 199-207. DOI: $10.1083 /$ jcb.200907161.

Peers C, Boyle JP, Scragg JL, Dallas ML, Al-Owais MM, Hettiarachichi NT, Elies J, Johnson E, Gamper N, Steele DS. 2015. Diverse mechanisms underlying the regulation of ion channels by carbon monoxide. British Journal of Pharmacology, 172: 1546-1556. DOI: 10.1111/bph.12760.

Piantadosi CA. 2008. Carbon monoxide, reactive oxygen signaling, and oxidative stress. Free Radical Biology and Medicine, 45: 562-569. DOI: 10.1016/j.freeradbiomed.2008.05.013. Pileggi A, Damaris Molano R, Berney T, Cattan P, Vizzardelli C, Oliver R, Fraker C, Ricordi C, Pastori RL, Bach FH, Inverardi L. 2001. Heme Oxygenase-1 Induction in Islet Cells Results in Protection from Apoptosis and Improved in Vivo Function after Transplantation. Diabetes, 50: 1983-1991. DOI: 10.2337/diabetes.50.9.1983. 
571 Prasad S, Tiwari M, Pandey AN, Shrivastav TG, Chaube SK. 2016. Impact of stress on oocyte

572

573

574

575

576

577

578

579

580

581

582

583

584

585

586

587

588

589

590

591

592

593

594

595

596

597

598

599

600

601

602

603

604

605

606

607

608

609

610 quality and reproductive outcome. Journal of Biomedical Science, 23. DOI: 10.1186/s12929-016-0253-4.

Qiu X, Yao B. 2017. Nrf2 regulates female germ cell meiosis initiation. Fertility and Sterility, 108: E153. DOI: 10.1016/j.fertnstert.2017.07.463.

Rizzo P, Raffone E, Benedetto V. 2010. Effect of the treatment with myo-inositol plus folic acid plus melatonin in comparison with a treatment with myo-inositol plus folic acid on oocyte quality and pregnancy outcome in IVF cycles. A prospective, clinical trial. European Review for Medical and Pharmacological Sciences, 14: 555-561.

Rybska M, Knap S, Jankowski M, Jesete M, Bukowska D, Antosik P, Nowicki M, Zabel M, Kempisty B, Jaśkowski JM. 2018. Cytoplasmic and nuclear maturation of oocytes in mammals - Living in the shadow of cells developmental capability. Medical Journal of Cell Biology, 6: 13-17. DOI: 10.2478/acb-2018-0003.

Ryter SW, Alam J, Choi AMK. 2006. Heme oxygenase-1/carbon monoxide: From basic science to therapeutic applications. Physiological Reviews, 86: 583-650. DOI: 10.1152/physrev.00011.2005.

Ryter SW, Choi AMK. 2016. Targeting heme oxygenase-1 and carbon monoxide for therapeutic modulation of inflammation. Translational Research, 167: 7-34. DOI: 10.1016/j.trs1.2015.06.011.

Ryter SW, Ma KC, Choi AMK. 2018. Carbon monoxide in lung cell physiology and disease. American Journal of Physiology-Cell Physiology, 314 (2): C211-C227. DOI: 10.1152/ajpcell.00022.2017.

Shimada M. 2012. Regulation of oocyte meiotic maturation by somatic cells. Reproductive Medicine and Biology, 11: 177-184. DOI: 10.1007/s12522-012-0130-0.

Shuhaibar LC, Egbert JR, Norris RP, Lampe PD, Nikolaev VO, Thunemann M, Wen L, Feil R, Jaffe LA. 2015. Intercellular signaling via cyclic GMP diffusion through gap junctions restarts meiosis in mouse ovarian follicles. Proceedings of the National Academy of Sciences of the United States of America, 112: 5527-5532. DOI: 10.1073/pnas.1423598112.

Schmitt A, Nebreda AR. 2002. Signalling pathways in oocyte meiotic maturation. Journal of Cell Science, 115: 2457-2459.

Soto-Herasa S, Paramio MT. 2020. Impact of oxidative stress on oocyte competence for in vitro embryo production programs. Research in Veterinary Science, 132: 342-350. DOI: 10.1016/j.rvsc.2020.07.013.

Tamura H, Jozaki M, Tanabe M, Shirafuta Y, Mihara Y, Shinagawa M, Tamura I, Maekawa R, Sato S, Takerani T, Takasaki A, Reiter RJ, Sugino N. 2020. Importance of Melatonin in Assisted Reproductive Technology and Ovarian Aging. International Journal of Molecular Sciences, 21: 1135. DOI: 10.3390/ijms21031135.

Tibullo D, Barbagallo I, Giallongo C, Vanella L, Conticello C, Romano A, Saccone S, Godos J, Di Raimondo F, Li Volti G. 2016. Heme oxygenase-1 nuclear translocation regulates bortezomibinduced cytotoxicity and mediates genomic instability in myeloma cells. 
611

612

613

614

615

616

617

618

619

620

621

622

623

624

625

626

627

628

629

630

631

632

633

634

635

636

637

638

639

640

641

642

643

644

645

646

Oncotarget, 7: 28868-28880. DOI: 10.18632/oncotarget.7563.

Tiwari M, Prasad S, Tripathi A, Pandey A, Singh A, Shrivastav T, Chaub S. 2016. Involvement of Reactive Oxygen Species in Meiotic Cell Cycle Regulation and Apoptosis in Mammalian Oocytes. Reactive Oxygen Species, 1: 110-116. DOI: 10.20455/ros.2016.817.

Vanhoutte L, Nogueira D, Dumortier F, De Sutter P. 2009. Assessment of a new in vitro maturation system for mouse and human cumulus-enclosed oocytes: Three-dimensional prematuration culture in the presence of a phosphodiesterase 3-inhibitor. Human Reproduction, 24: 1946-1959. DOI:10.1093/humrep/dep104.

Wang S, He G, Chen M, Zuo T, Xu W, Liu X. 2017. The Role of Antioxidant Enzymes in the Ovaries. Oxidative Medicine and Cellular Longevity, 2017: 4371714. DOI: 10.1155/2017/4371714.

Wang Y, Chen K, Li C, Li L, Wang G. 2018. Heme oxygenase 1 regulates apoptosis induced by heat stress in bovine ovarian granulosa cells via the ERK1/2 pathway. Journal of Cellular Physiology, 234: 3961-3972. DOI: 10.1002/jcp.27169.

Wang Y, Yang C, Elsheikh NAH, Li C, Yang F, Wang G, Li L. 2019. HO-1 reduces heat stress-induced apoptosis in bovine granulosa cells. Aging, 11: 5535-5547. DOI: 10.18632/aging.102136.

Wegiel B, Chin BY, Otterbein LE. 2008. Inhale to survive, cycle or die? Carbon monoxide and cellular proliferation. Cell Cycle, 7: 1379-1384. DOI: 10.4161/cc.7.10.5948.

Weston CR, Davis RJ. 2007. The JNK signal transduction pathway. Current Opinion in Cell Biology, 19: 142-149. DOI: 10.1016/j.ceb.2007.02.001.

Wu L, Wang R. 2005. Carbon monoxide: Endogenous production, physiological functions, and pharmacological applications. Pharmacological Reviews, 57: 585-630. DOI: 10.1124/pr.57.4.3.

Xie Y, Wu B, Jin Y, Zhang A, Sun X, Zhang X, Gao X, Dong R, Li H, Gao J. 2018. Oocytespecific deletion of $\mathrm{Gs} \alpha$ induces oxidative stress and deteriorates oocyte quality in mice. Experimental Cell Research, 370: 579-590. DOI: 10.1016/j.yexcr.2018.07.023.

Yu K, Wang R, Li M, Sun T, Zhou Y, Li Y, Sun L, Zhang B, Lian Z, Xue S, Liu Y, Deng S. 2019. Melatonin Reduces Androgen Production and Upregulates Heme Oxygenase-1 Expression in Granulosa Cells from PCOS Patients with Hypoestrogenia and Hyperandrogenia. Oxidative Medicine and Cellular Longevity, 2019: 8218650. DOI: $10.1155 / 2019 / 8218650$.

Zenclussen ML, Jensen F, Rebelo S, El-Mousleh T, Casalis PA, Zenclussen AC. 2012. Heme Oxygenase-1 Expression in the Ovary Dictates a Proper Oocyte Ovulation, Fertilization, and Corpora Lutea Maintenance. American Journal of Reproductive Immunology, 67: 376382. DOI: 10.1111/j.1600-0897.2011.01096.x. 


\section{Table $\mathbf{1}$ (on next page)}

Expression levels of $\mathrm{HO}-1$ and $\mathrm{HO}-2$ mRNA and protein in porcine oocytes during meiotic maturation.

The expression level of mRNA was analyzed by RT-PCR in oocytes at GV, MI, and MIl stages. The relative mRNA level was normalized to GAPDH and relative to oocytes at the GV stage. The amount of mRNA was calculated using the arithmetic equation $2^{-\Delta \Delta C T}$ according to the $\mathrm{Ct}$ method. The data are presented as mean \pm SEM relatively to mean mRNA level in oocytes at the GV stage. The expression of HO-1 and HO-2 proteins is assessed by the immunocytochemical method in oocytes at the GV, MI, and MII stage. The date is expressed as mean \pm SEM relative to the mean signal intensity of HO- 1 or HO-2 in oocytes at the GV stage. The amount of mRNA and HO-1 and HO-2 proteins between different meiotic maturation stages were not significant. 


\begin{tabular}{l|cccc}
\hline \multirow{2}{*}{ Culture period } & HO-1 & HO-2 & HO-1 & HO-2 \\
\cline { 2 - 5 } & 1.00 & 1.00 & 1.00 & 1.00 \\
\hline 0 hours (GV) & $0.61 \pm 0.131$ & $0.85 \pm 0.086$ & $1.05 \pm 0.039$ & $0.85 \pm 0.030$ \\
24 hours (MI) & $1.31 \pm 0.308$ & $0.98 \pm 0.073$ & $1.10 \pm 0.028$ & $0.94 \pm 0.031$ \\
\hline
\end{tabular}

1

2 


\section{Figure 1}

Localization of heme oxygenase-1 (HO-1) in porcine oocytes during meiotic maturation.

HO-1 is shown in green (FITC), chromatin is shown in blue (DAPI), magni-fied 400x; GV germinal vesicle, MI - metaphase I, MII - metaphase II.

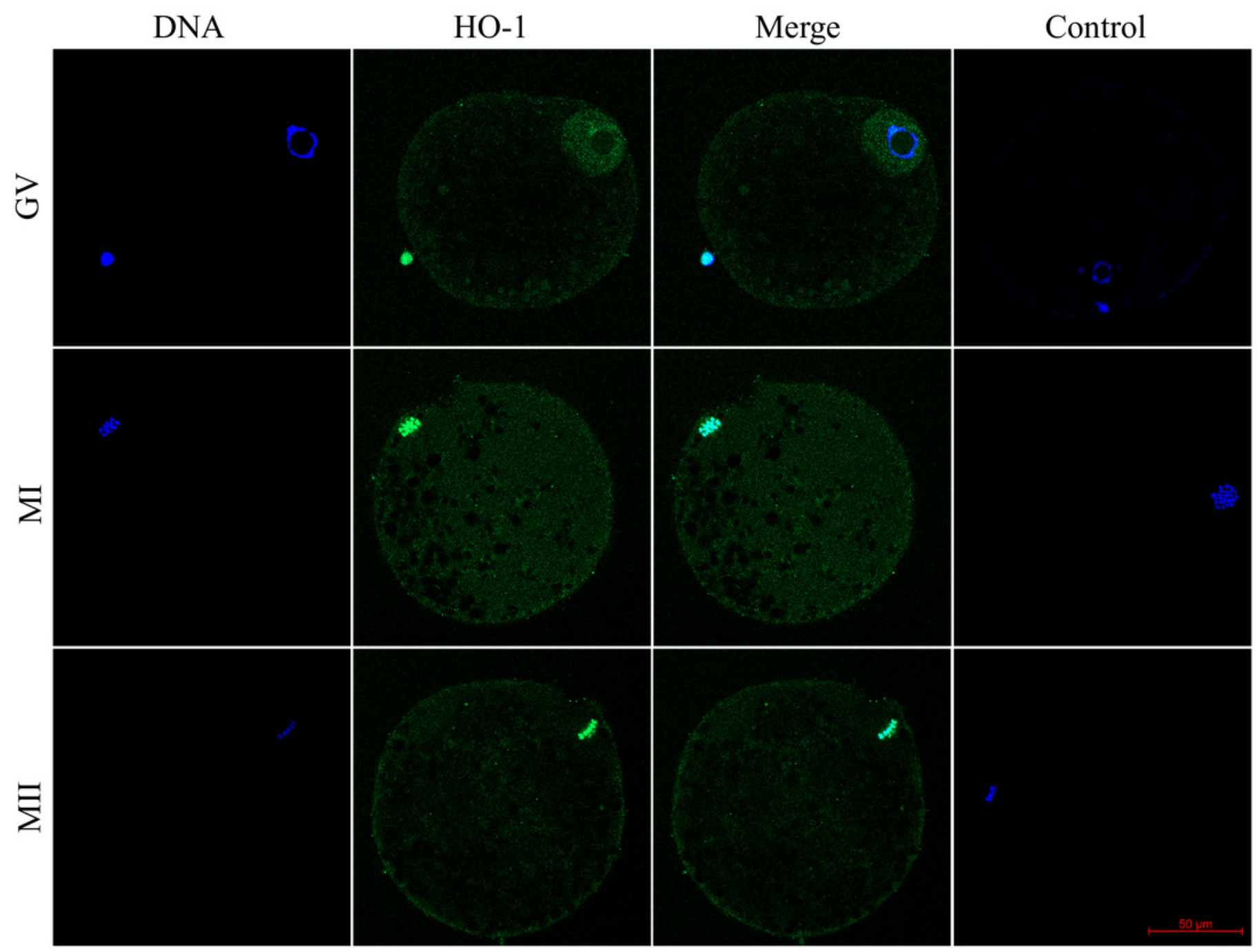




\section{Figure 2}

Localization of heme oxygenase-2 (HO-2) in porcine oocytes during meiotic maturation. HO-2 is shown in green (FITC), chromatin is shown in blue (DAPI), magni-fied 400x; GV germinal vesicle, MI - metaphase I, MII - metaphase II.

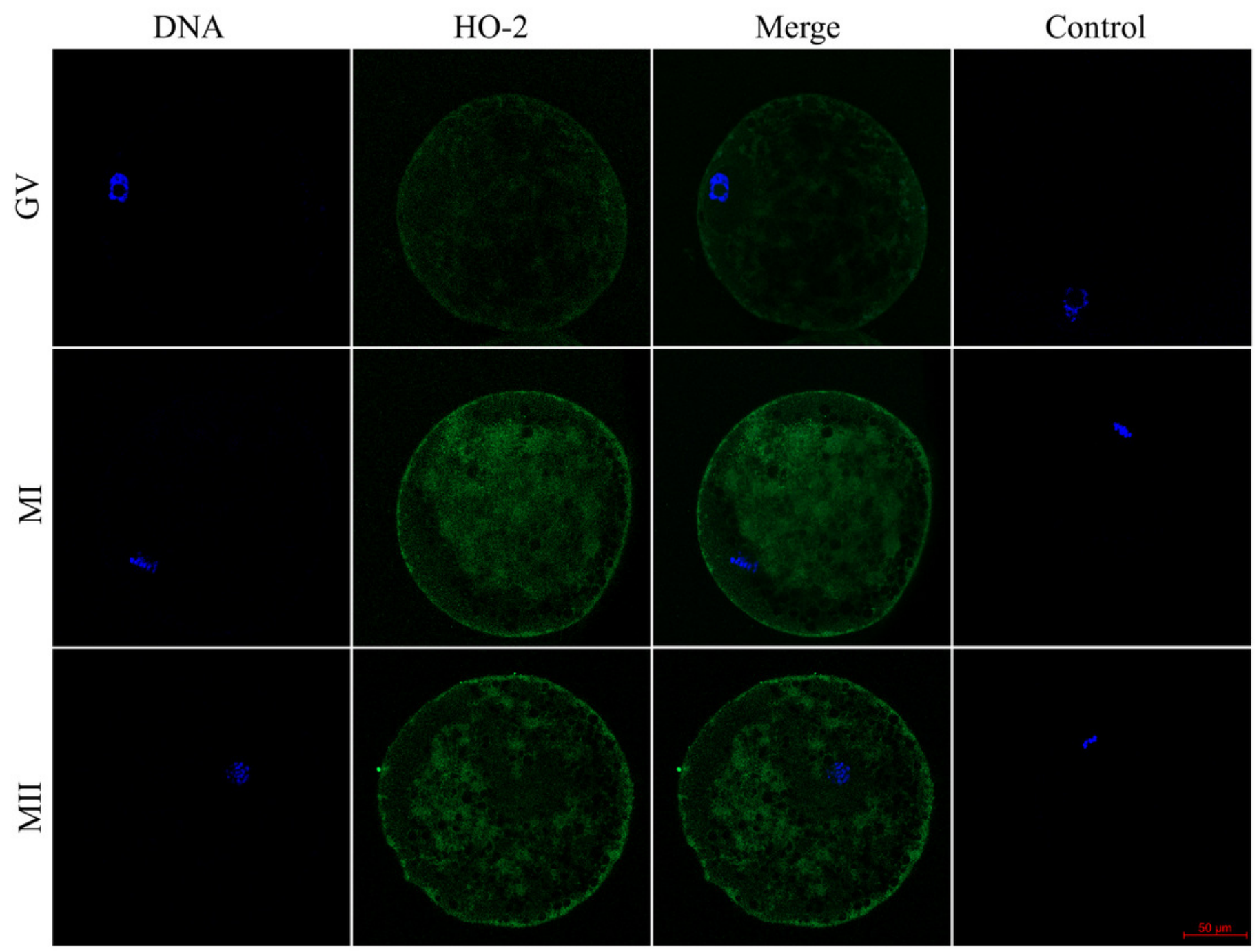




\section{Figure 3}

Effect of HO inhibitor ZnPP-IX on the meiotic maturation of porcine oocytes.

The control group was cultured in a culture medium containing DMSO. Experimental groups were cultured in a culture medium containing ZnPP-IX at concentrations of 1, 2.5, 5, 10, or 25 $\mu \mathrm{M}$. The date is expressed as mean $\pm \mathrm{SEM}$. Stages of nuclear maturation were evaluated as degenerate (Deg), germinal vesicle (GV), metaphase I (MI), and metaphase II (MII). Superscripts ${ }^{\text {ab }}$ shows a statistically significant difference in the proportion of oocytes at the MIl stage $(P<0.05)$. Superscripts '" shows a statistically significant difference in the proportion of oocytes at the Ml stage $(P<0.05)$. Superscripts ${ }^{12}$ shows a statistically significant difference in the proportion of oocytes at the GV stage $(P<0.05)$. Superscripts ${ }^{* * *}$ shows a statistically significant difference in the proportion of Deg oocytes $(P<0.05)$. 


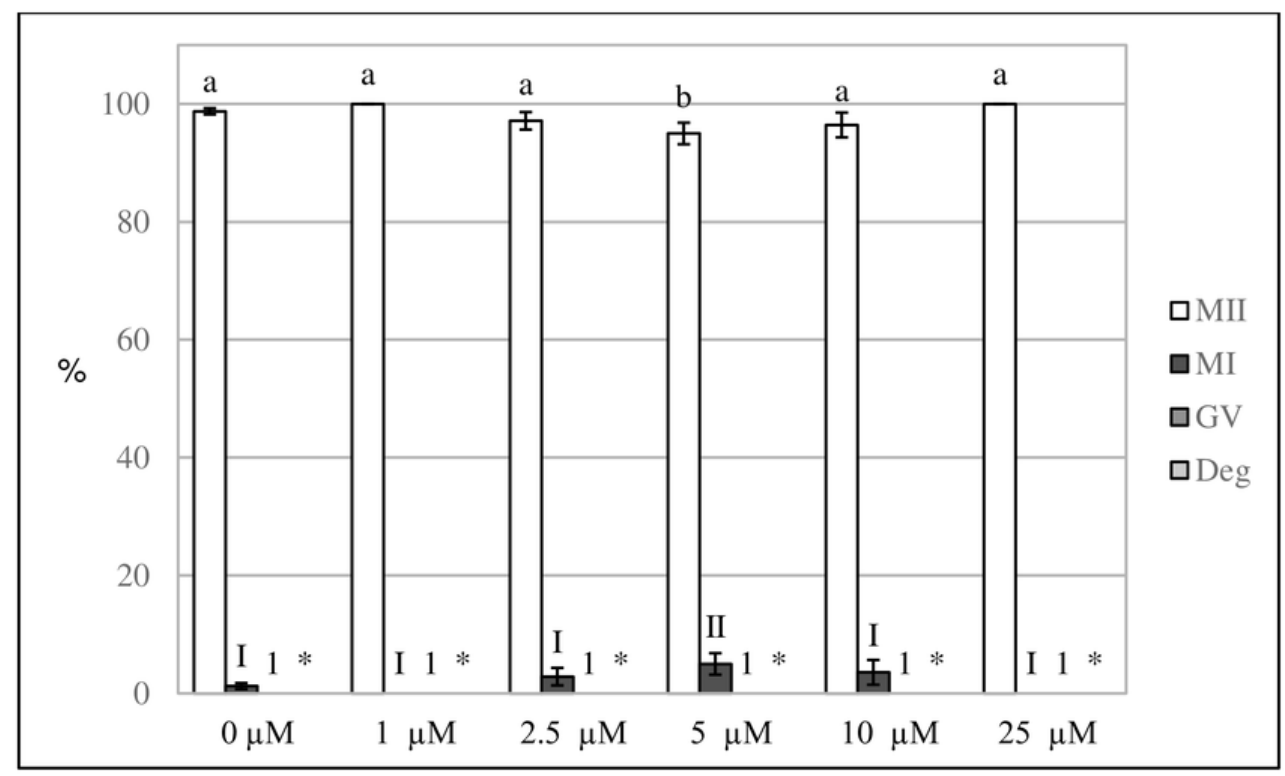




\section{Figure 4}

Effect of CORM-2 (A) and CORM-A1 (B) on meiotic maturation of porcine oocytes.

The control group was cultured in a culture medium containing an inactive form of CORM (iCORM-2 or iCORM-A1). Experimental groups were cultured in a culture medium containing CORM-2 or CORM-A1 at concentrations of 5, 25, 50, and $100 \mu \mathrm{M}$. The date is expressed as mean \pm SEM. Stages of nuclear maturation were evaluated as degenerate (Deg), germinal vesicle (GV), metaphase I (MI), and metaphase II (MII). Superscripts ${ }^{\text {ab }}$ shows a statistically significant difference in the proportion of oocytes at the MII stage $(P<0.05)$. Superscripts "IIIII shows a statistically significant difference in the proportion of oocytes at the MI stage ( $P$ $<0.05$ ). Superscripts ${ }^{12}$ shows a statistically significant difference in the proportion of oocytes at the GV stage $(P<0.05)$. Superscripts ${ }^{* * *}$ shows a statistically significant difference in the proportion of Deg oocytes $(\mathrm{P}<0.05)$. 


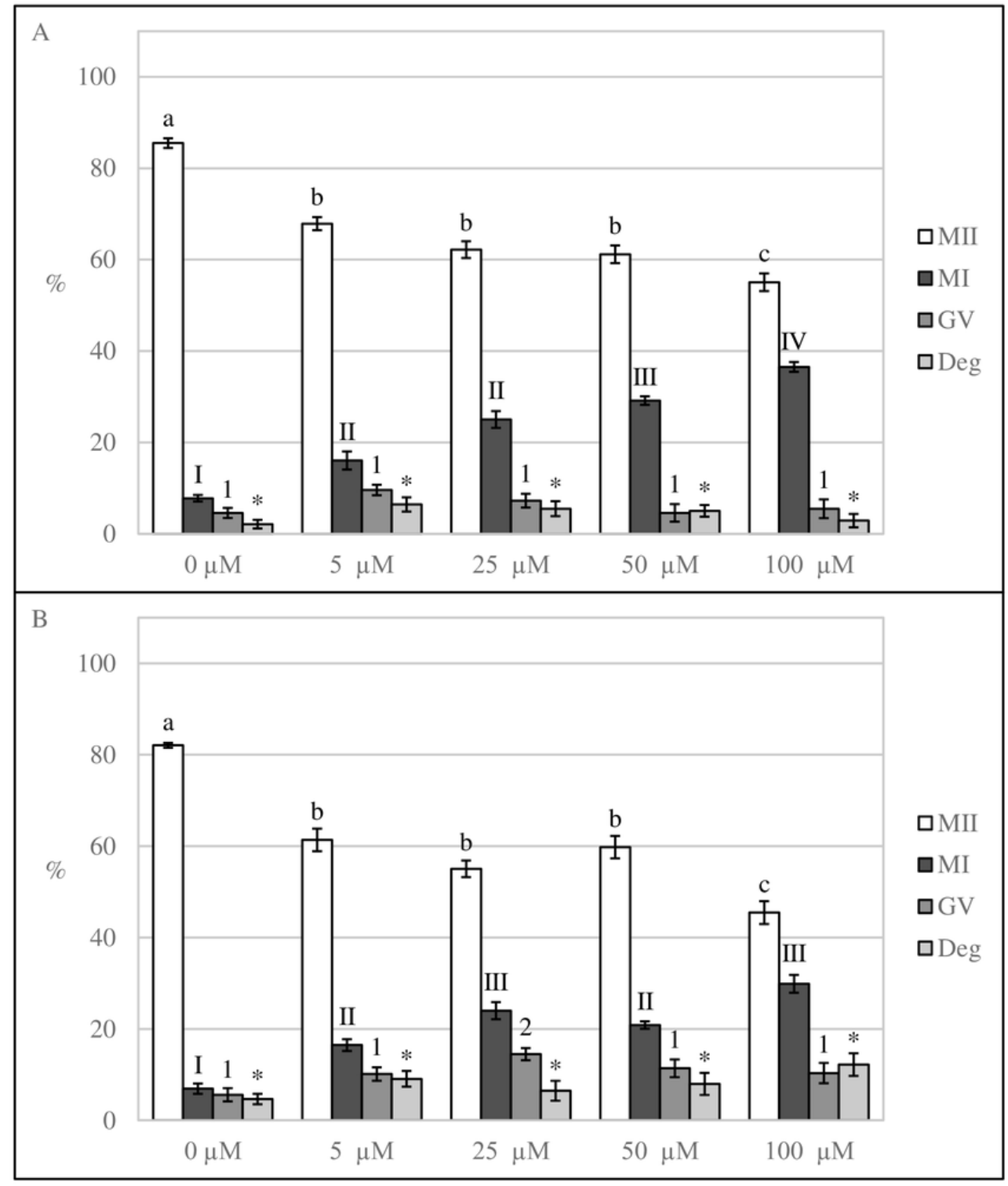




\section{Figure 5}

Effect of CORM-2 on prolonged cultivation of porcine oocytes.

The control group was cultured in a culture medium containing an inactive form of CORM (iCORM-2). Experimental groups were cultured in a culture medium containing CORM-2 at concentrations of $5,25,50$, and $100 \mu \mathrm{M}$. The date is expressed as mean \pm SEM. Stages of nuclear maturation were evaluated as degenerate (Deg), germinal vesicle (GV), metaphase I (MI), and metaphase II (MII). Superscripts ${ }^{\text {ab }}$ shows a statistically significant difference in the proportion of oocytes at the MII stage $(P<0.05)$. Superscripts '" shows a statistically significant difference in the proportion of oocytes at the MI stage $(P<0.05)$. Superscripts ${ }^{12}$ shows a statistically significant difference in the proportion of oocytes at the GV stage (P $<0.05)$. Superscripts ${ }^{* * *}$ shows a statistically significant difference in the proportion of Deg oocytes $(P<0.05)$. 


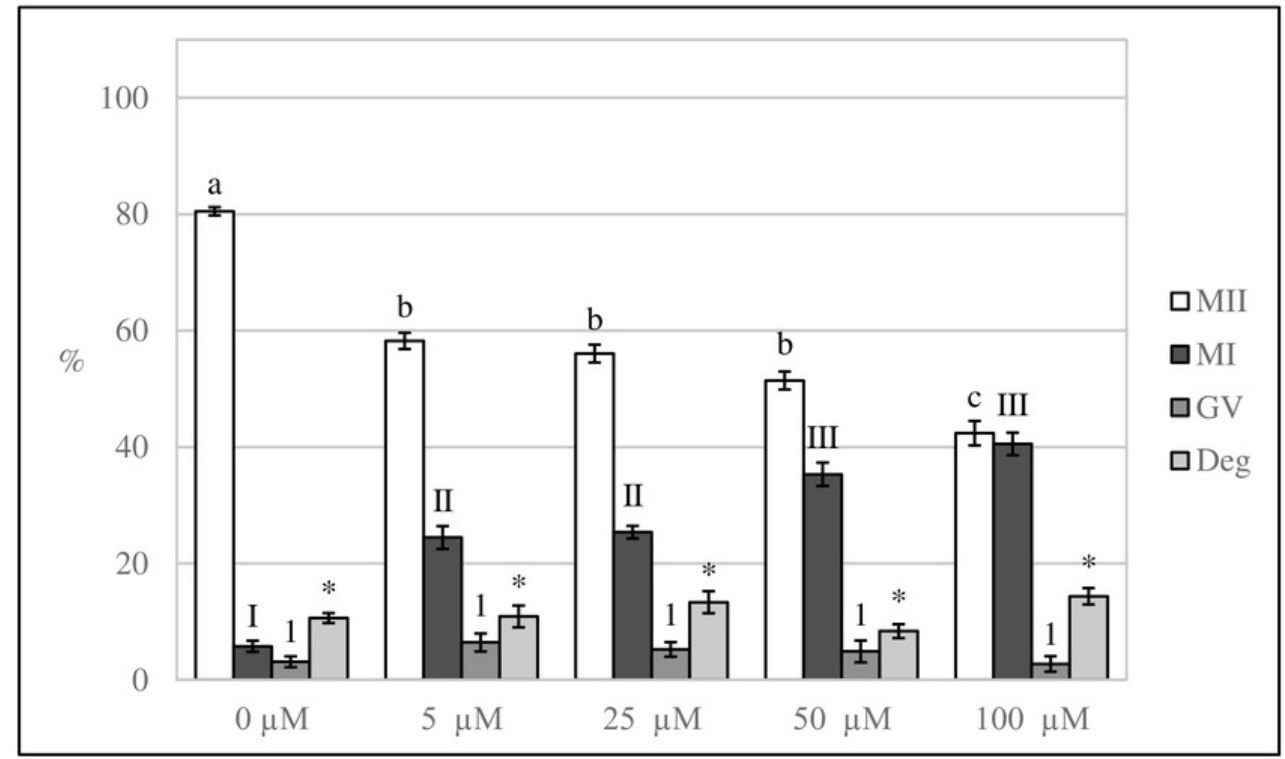




\section{Figure 6}

Assessment of reactive oxygen species (ROS) production in the porcine oocyte during meiotic maturation.

The control group demonstrates the level of non-specific signal intensity in oocytes without treatment with 2,7-dichlorodihydrofluorescein diacetate method. The group $0 \mu \mathrm{M}$ were cultivated in culture medium supplemented with iCORM-2. The experimental groups were a cultivated in culture medium containing CORM-2 at various concentrations. The level of ROS production was detected using the 2,7-dichlorodihydrofluorescein diacetate (green). Magnified 200x. 
Control

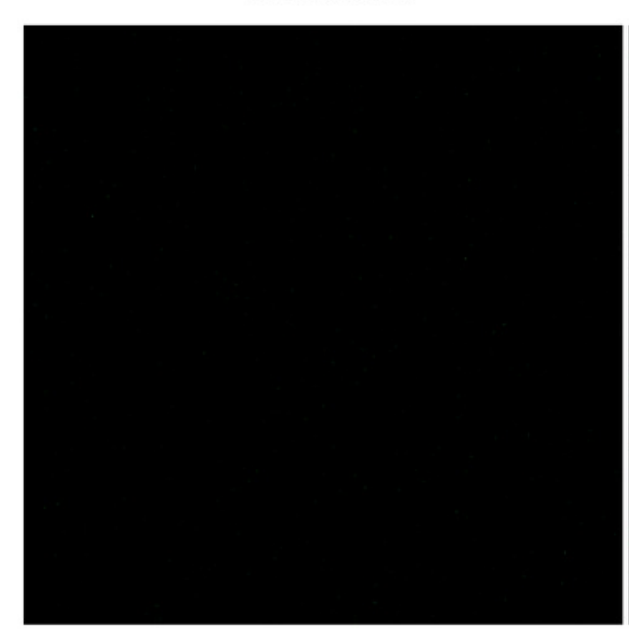

$25 \mu \mathrm{M}$
$0 \mu \mathrm{M}$

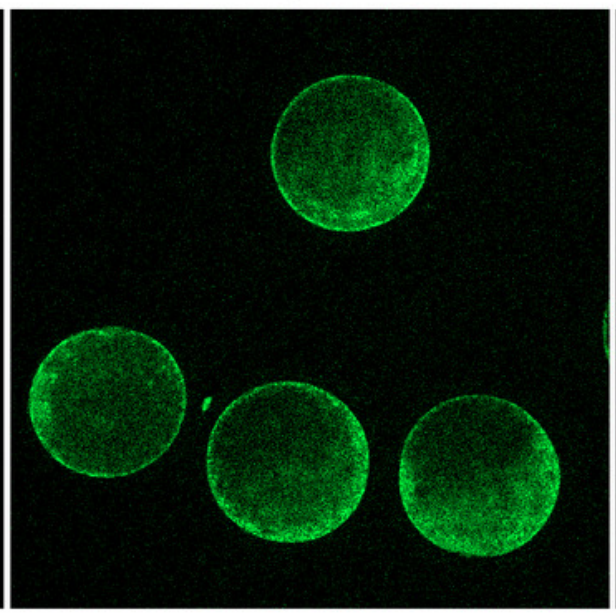

$50 \mu \mathrm{M}$
$5 \mu \mathrm{M}$

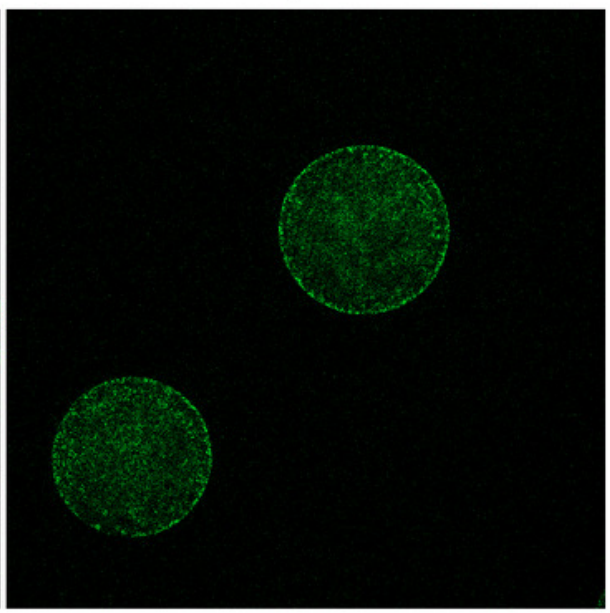

$100 \mu \mathrm{M}$
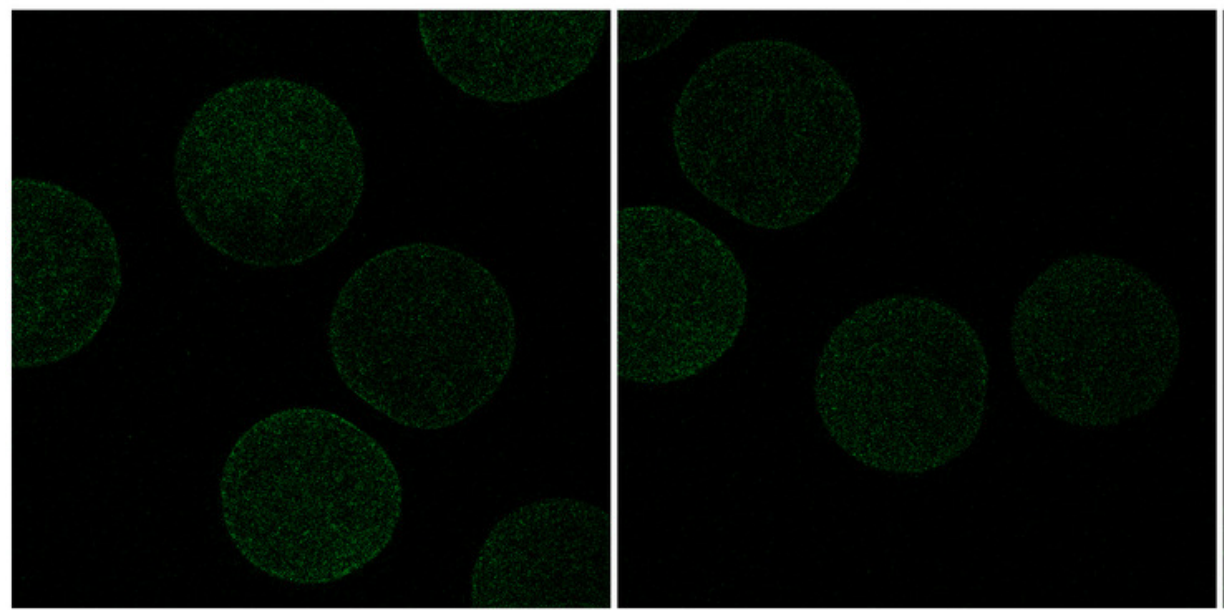


\section{Figure 7}

Effect of CORM-2 on ROS level in porcine oocytes during meiotic maturation.

The control group was cultured in a culture medium containing an inactive form of CORM (iCORM-2). Experimental groups were cultured in a culture medium containing CORM-2 at concentrations of 5, 25, 50, and $100 \mu \mathrm{M}$. The level of ROS in porcine oocytes is expressed as the mean signal intensity of 2.7-dichlorodihydrofluorescindiacetate and is relative to the signal intensity in oocytes at the GV stage. Bars show the mean \pm SEM. Superscripts ${ }^{a b c}$ shows a statistically significant difference in the level of ROS compared to the control group $(\mathrm{P}<0.05)$. 


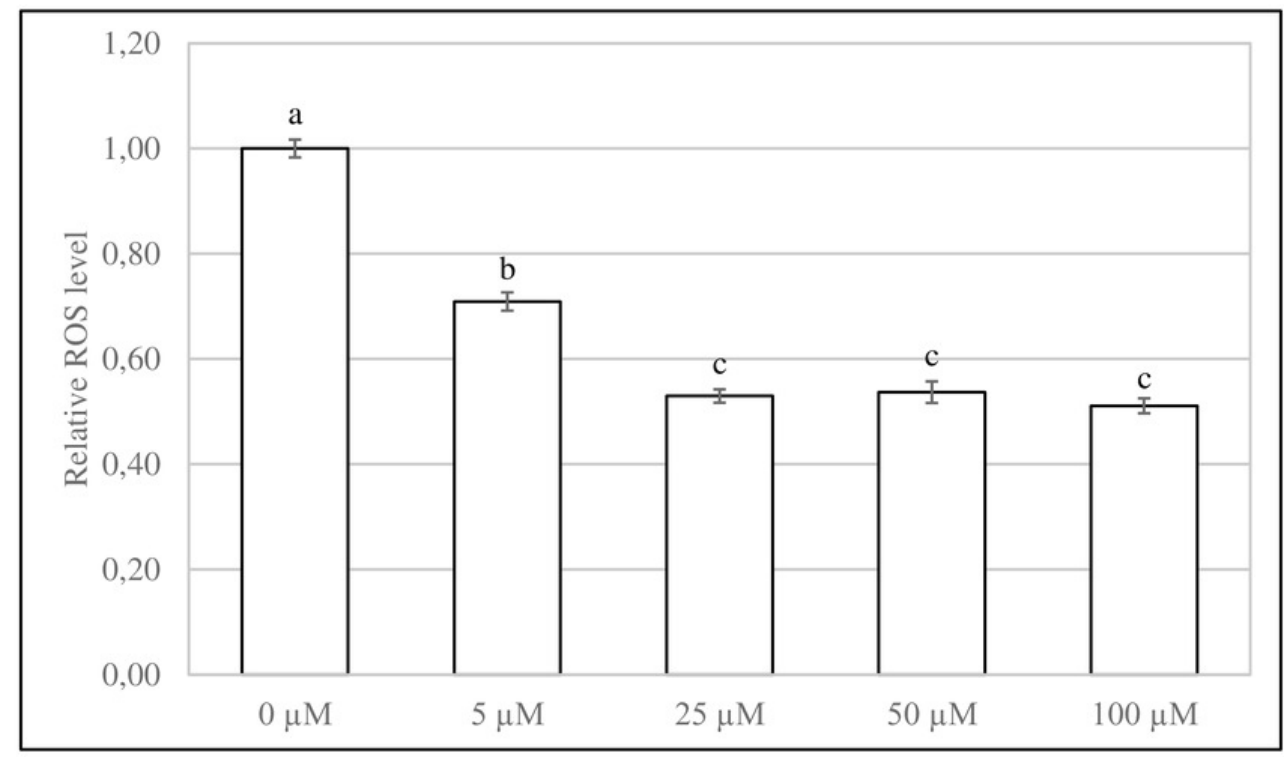

